442

THE INFLUENCE OF HMGB1 GENE (RS41369348) POLYMORPHISM ON THE SUSCEPTIBILITY AND CLINICAL FEATURES OF PATIENTS WITH IGAV

Mateja Batnozic Varga* , Mario Sestan, Jasenka Wagner, Kristina Crkvenac, Nastasia Kifer, Marijan Frkovic, Laura Stefinovec, Danica Grguric, Silvija Puseljic, Marija Jelusic. University Hospital Centre Osijek, Department of Paediatrics, Josip Juraj Strossmayer University of Osijek, Faculty of Medicine

\subsection{6/archdischild-2021-europaediatrics.442}

IgA vasculitis (IgAV) or Henoch-Schönlein's purpura is the most prevalent systemic small vessel vasculitis in childhood, in which pathogenesis environmental and genetic factors play role. From genetic factors different studies proven that genes located inside the HLA region and gene polymorphisms located outside the HLA region contribute to the onset and different clinical features of IgAV. The aim of this study was to investigate the role of single nucleotide polymorphism (SNP)- rs41369348 (delT; chr13:30467220-30467227 (GRCh38.p12)) for high mobility group box-1 (HMGB1) gene in the susceptibility and clinical features of patients fulfilling classification criteria for IgAV.

In this study, we included 76 children with IgAV and 150 age- and sex-matched healthy controls without any history of autoimmune disease.

After extracting genomic DNA from the whole peripheral blood, genotyping was carried by real-time PCR method using TaqMan SNP genotyping assays. Clinical data and laboratory parameters were collected for all IgAV patients.

The normal $\mathrm{T} / \mathrm{T}$ genotype was found in $83 \%$ of the IgAV patients and $91 \%$ of the control group. Heterozygous T/delT genotype was detected in $11 \%$ and $8 \%$ of the patient group and healthy controls, respectively. Homozygous mutant delT genotype was found in only 1 IgAV patient.

Although there was higher frequency of heterozygous T/ delT genotype of this gene polymorphism in IgAV group compared to control group, no genotype difference between those two groups was observed. No statistically significant differences in genotype nor allele were disclosed when patients with different IgAV clinical features were compared.

Accordingly to our study, the HMGB1 gene polymorphism rs41369348 was not linked to increased susceptibility to childhood IgAV, its severity nor different clinical manifestations.

SUPPORT: Croatian Science Foundation project IP-2019-048822

\section{DIFFERENT HISTOLOGICAL CLASSIFICATIONS FOR IGA VASCULITIS NEPHRITIS - WHICH ONE HAS THE BEST ASSOCIATION WITH THE DISEASE OUTCOME?}

Nastasia Kifer*, Mario Sestan, Martina Held, Marijana Coric, Stela Bulimbasic, Teresa Giani, Neil Martin, Sasa Srsen, Ana Gudelj Gracanin, Domagoj Kifer, Merav Heshin, Angelo Ravelli, Rolendo Cimaz, Seza Ozen, Alenka Gagro, Marija Jelusic. University Hospital Centre Zagreb, Department of Paediatrics, University of Zagreb School of Medicine, Zagreb, Croatia

\subsection{6/archdischild-2021-europaediatrics.443}

Introduction IgA vasculitis nephritis (IgAVN) is almost the only cause of morbidity and mortality among children suffering from this most common childhood-vasculitis. Several histological classifications are used in the analysis of renal biopsy findings in IgAVN, but it remains unknown which one is the best predictor of severity and disease outcome.
The aim was to compare the four most commonly used histologic classifications for IgAVN and to establish which variables of each histological classification have the strongest association with unfavorable outcome.

The cross-sectional study included 74 patients with IgAVN (diagnosed by EULAR/PRES/PRINTO criteria) and available renal biopsy specimens for analysis using the four histological classifications for IgAVN (the International Study of Kidney Disease in Children (ISKDC) classification, the Oxford classification, the Haas histologic classification of IgA nephropathy and the modified semi-quantitative classification (SQC), developed by Koskela et al.). The clinical outcome was defined through four categories, graded according to the modified classification of Counahan (physical examination, hematuria, proteinuria, urine albumin-tocreatinine ratio, hypertension and eGFR). The linear relationships between outcome and histological classifications were analysed using ordinal regressions using the first-order of polynomial orthogonal contrasts.

The SQC classification proved to be the best, reducing the deviation (of the model-predicted outcome value from the observed value) by $9.5 \%(\chi 21=13,89, \mathrm{p}<0,001)$, followed by the Oxford classification with a deviation reduction of $8.0 \%$ $(\chi 21=11,76, p=0,001)$, then the ISKDC classification with a decrease in deviation of $3.3 \% \quad(\chi 21=4,89, p=0,027)$. The worst was the Haas classification with a decrease in deviation of $2.1 \%(\chi 21=3,06, p=0,080)$.

Analysis of individual variables of Oxford and SQC classifications showed that with increasing values in the variables of interstitial fibrosis (t66 $=3,23, \mathrm{p}=0,002)$, tubular atrophy (t66 $=2,94, p=0,005)$ and tubular dilatation ( $t 66=2,40$, $\mathrm{p}=0,019)$ in the SQC classification, and endocapillary hypercellularity (t66 $=3,14, p=0,003)$ and crescents (t66 $=2,07$, $\mathrm{p}=0,043)$ in the Oxford classification the outcome worsens.

This study showed that the SQC classification has the strongest association with the IgAVN severity and outcome. Although crescents on renal biopsy were considered the most important outcome indicators, our study suggests that tubulointerstitial changes could be more important as predictors of poor outcome. Interstitial and renal tubules changes should be further explored in order to have better predictive values of IgAVN outcome and to be incorporated into existing or new classifications, on the basis of which guidelines for the treatment of patients would be developed.

SUPPORT: Croatian Science Foundation project IP-2019-048822 .

\section{THE IMPACT OF SYSTEMIC IMMUNOMODULATORY THERAPY ON THE INTRAOCULAR INFLAMMATION AND THE NEED FOR TOPICAL GLUCOCORTICOID THERAPY IN PATIENTS WITH JUVENILE IDIOPATHIC ARTHRITIS- ASSOCIATED UVEITIS}

Mario Sestan*, Barisic Kutija Marija, Sestan Mario, Peric Sanja, Kifer Nastasia, Ivkic Petra Kristina, Galiot Delic Martina, Jandrokovic Sonja, Held Martina, Frkovic Marijan, Jelusic Marija, Vukojevic Nenad. University Hospital Centre Zagreb, Department of Paediatrics, University of Zagreb School of Medicine

\subsection{6/archdischild-2021-europaediatrics.444}

Juvenile idiopathic arthritis associated uveitis (JIA-U) is the most common and potentially most destructive extraarticular manifestation of JIA. The aim of this research was to 
determine the need for topical glucocorticoid therapy (TGC) in patients with JIA-U on systemic biological therapy in comparison to patients treated with methotrexate (MTX) only.

We have conducted longitudinal observational study with JIA-U patients in whom systemic immunomodulatory treatment (IMT: biologics and/or MTX) was introduced and who were followed at least 3 months in the period between 2011 and 2017. The data about the number of cells in the anterior chamber (AC) according to Standardization of Uveitis Nomenclature (SUN) Working Group criteria, about TGC and systemic therapy and JIA complications were collected on each examination. Generalized linear mixed models were used to analyze the relationships between treatment with biologics, MTX, TGC and the grade of inflammation in AC according to SUN criteria.

38 JIA-U patients (69 eyes) with median (range) age of 4.9 (2-15) years and follow up period of 209 (19-381) weeks were included. There were a total of 1205 examinations. At the first examination JIA-U was detected in $16(42.1 \%)$ of patients, $59(79.7 \%)$ of the eyes had $\leq 1+$ cells in the AC, and in $19(50 \%)$ of JIA-U patients complications were already present. MTX was introduced in 23 (60.5\%) JIA-U patients before the inclusion in the study, $8(21 \%)$ has already received biologics, while in $4(10.5 \%)$ prior systemic glucocorticoids were also used. Until the end of the study, all patients received MTX and 40\% JIA-U were treated with biologics. The average number of TGC doses decreased from 3.74 at baseline to 0.72 in the 48 th month.

After Friedman and the post hoc test a significant difference in the daily doses of TGC could be seen from the 12th month after application of systemic IMT. The number of daily doses of TGC as well as the degree of inflammation in AC per eye decreased over time. Using generalized linear mixed models it was shown that the treatment with biologics, but not with MTX and systemic glucocorticoids, was associated with lower intensity of TGC therapy.

Treatment with biologics and systemic glucocorticoids, but not with MTX, were associated with lower degree of inflammation in AC.

The results showed that the application of systemic biological therapy may result in less intensive TGC therapy, resulting in glucocorticoid-sparing potential, and reducing intraocular inflammation.

\section{DETERMINING THE EFFECTIVENESS OF SYSTEMIC IMMUNOMODULATORY THERAPY IN THE TREATMENT OF PATIENTS WITH JUVENILE IDIOPATHIC ARTHRITIS ASSOCIATED UVEITIS DEPENDING ON THE CHOSEN OUTCOME MEASURES}

Barisic Kutija Marija, Sestan Mario*, Peric Sanja, Kifer Nastasia, Ivkic Petra Kristina, Galiot Delic Martina, Knezevic Josip, Held Martina, Frkovic Marijan, Jelusic Marija, Vukojevic Nenad. University Hospital Centre Zagreb, Department of Paediatrics, University of Zagreb School of Medicine

\subsection{6/archdischild-2021-europaediatrics.445}

The number of patients with juvenile idiopathic arthritis associated uveitis (JIA-U) who need systemic immunomodulatory treatment (IMT) for disease control is small. Variabilities in the patient selection and the results on the effectiveness of IMT make it difficult to compare studies. We aimed to show on the same sample of JIA-U patients how different the obtained levels of therapy efficacy are depending on the selected outcomes.

The longitudinal observational study with JIA-U patients treated with IMT was conducted at University Hospital Centre Zagreb in the period from 2011 to 2017.

We included 38 JIA-U patients aged 2 to 15 years and 69 eyes (7 patients had unilateral JIA-U). At baseline 46 (66.7\%) eyes had grade $\leq 1+$ of inflammation in anterior chamber (AC) according to Standardization of Uveitis Nomenclature (SUN) Working Group criteria, 11 (15.9\%) had grade 2+, 3 (4.4\%) had grade $3+.23$ children $(60.5 \%)$ had already received methotrexate (MTX), 8 (21.0\%) biologics, 4 $(10.5 \%)$ systemic glucocorticoids (GC). Topical glucocorticoids (TGC) in the form of drops were used in $61(88.4 \%)$ eyes with a median of 4 daily doses, GC ointment in $75.4 \%$ of the eyes with a median of 1 dose. Until the end of the follow-up, all children received MTX, and 40\% of them biologics. The results of the effectiveness of IMT are presented according to the reduction of the need for TGC and achieved level of inflammation in AC. In the first 12 months, among JIA-U patients treated with both biologics and MTX, in $65 \%$ of eyes there was no need for TGC therapy, in the 48th month in 50\% of eyes, while the rest required 1-2 daily doses of TGC. At the end of the first year, with MTX and biological therapy $75 \%$ of eyes had grade 0 of inflammation and in 48th month $61.1 \%$. In the 12 th month in $75 \%$ of eyes a grade 0 of inflammation was achieved with $\leq 2$ doses of TGC, and in the 48th month in $61.1 \%$. According to milder criteria, in the 12th month $90 \%$ of the eyes have a degree of inflammation $\leq 0,5+$ with $\leq 2$ doses of TGC, and in the 48th month all patients achieved this goal.

It was shown that the results of treatment outcomes during follow-up largely depend on the selected outcome measures. This will be important for future research because setting different limits can lead to a more favorable outcome.

\section{SINGLE NUCLEOTIDE POLYMORPHISMS OF GENES HMGB1 AND AGER AND ITS ASSOCIATION WITH CLINICAL FEATURES OF IGA VASCULITIS}

Martina Held*, Mateja Batnozic Varga, Mario Sestan, Matej Sapina, Nastasia Kifer, Danica Grguric, Kristina Crkvenac Gornik, Marijan Frkovic, Nena Arvaj, Jasenka Wagner, Marija Jelusic. University Hospital Centre Zagreb, University of Zagreb School of Medicine

\subsection{6/archdischild-2021-europaediatrics.446}

IgA vasculitis (IgAV) is a desease in which genetic background also plays an important role. Some small studies have indicated the importance of variants in various non-HLA genes in the manifestation of different disease phenotypes. The aim of this research was to investigate single nucleotide polymorphisms (SNPs) of genes HMGB1 and AGER encoding for high mobility group box-1 (HMGB1) and receptor for advanced glycation endproducts (RAGE), both acting as mediators of inflammation, in the susceptibility and clinical features of patients with $\mathrm{IgAV}$.

HMGB1 and RAGE gene polymorphisms were genotyped using a real-time polymerase chain reaction. The presence and frequency of polymorphisms in HMGB1 (rs2249825, rs1045411, rs1060348, rs1412125 and rs41369348) and RAGE (rs1800625, rs1800624, rs2070600 and rs3134940) were determined. Clinical data were collected from database 